\title{
CARMENES: Blue Planets Orbiting Red Dwarfs
}

\section{A. Quirrenbach ${ }^{1}$, P. J. Amado ${ }^{2}$, J. A. Caballero ${ }^{3}$, H. Mandel ${ }^{1}$, R. Mundt ${ }^{4}$, A. Reiners ${ }^{5}$, I. Ribas ${ }^{6}$, M. A. Sánchez Carrasco ${ }^{2}$, W. Seifert ${ }^{1}$, M. Azzaro ${ }^{7}$, D. Galadí í and the CARMENES Consortium ${ }^{1,2,3,4,5,6,7,8,9,10,11}$}

${ }^{1}$ Landessternwarte, Zentrum für Astronomie der Universität Heidelberg, Königstuhl 12, D-69117 Heidelberg, Germany

${ }^{2}$ Instituto de Astrofísica de Andalucía (CSIC), Glorieta de la Astronomía s/n, E-18008 Granada, Spain

${ }^{3}$ Centro de Astrobiología (CSIC-INTA), Campus ESAC, PO Box 78, E-28691 Villanueva de la Cañada, Madrid, Spain

${ }^{4}$ Max-Planck-Institut für Astronomie, Königstuhl 17, D-69117 Heidelberg, Germany

${ }^{5}$ Institut für Astrophysik (IAG), Friedrich-Hund-Platz 1, D-37077 Göttingen, Germany

${ }^{6}$ Institut de Ciències de l'Espai (CSIC-IEEC), Campus UAB, Facultat Ciències, Torre C5 parell - 2a planta, E-08193 Bellaterra, Barcelona, Spain

${ }^{7}$ Calar Alto Observatory (MPG-CSIC), Centro Astronómico Hispano-Alemán, Jesús Durbán Remón, 2-2, E-04004 Almería, Spain

${ }^{8}$ Departamento de Astrofísica, Facultad de Física, Universidad Complutense de Madrid, E-28040 Madrid, Spain

${ }^{9}$ Thüringer Landessternwarte Tautenburg, Sternwarte 5, D-07778 Tautenburg, Germany

${ }^{10}$ Instituto de Astrofísica de Canarias, Vía Láctea s/n, E-38205 La Laguna, Tenerife, Spain, and Dept. Astrofísica, Universidad de La Laguna (ULL), E-38206 La Laguna, Tenerife, Spain

${ }^{11}$ Hamburger Sternwarte, Gojenbergsweg 112, D-21029 Hamburg, Germany

\begin{abstract}
CARMENES (Calar Alto high-Resolution search for $\mathbf{M}$ dwarfs with Exo-earths with Near-infrared and optical Echelle Spectrographs) will conduct a radial-velocity survey of $\sim 300 \mathrm{M}$ dwarfs with the $3.5 \mathrm{~m}$ telescope at the Calar Alto Observatory. The CARMENES instrument is currently under construction; it consists of two independent échelle spectrographs, which together cover the wavelength range $0.55-1.7 \mu \mathrm{m}$ at a spectral resolution of $R=82,000$. The spectrographs and the fiber input are designed with a goal of $1 \mathrm{~m} / \mathrm{s}$ radial velocity precision using simultaneous calibration with emission-line lamps.
\end{abstract}

Keywords. instrumentation: spectrographs, techniques: radial velocities, stars: late-type, (stars:) planetary systems

\section{Introduction}

M-type stars span the mass range of $0.1-0.6 M_{\odot}$, and therefore obtaining statistics of planetary system occurrence and architecture for these stars is of great importance for understanding the physics of planet formation and evolution, and its dependence on stellar host mass. Radial-velocity searches for planets around M dwarfs benefit from a large signal and a short orbital period of planets in the habitable zone, which provides an opportunity for finding habitable planets in the Solar neighborhood. However, the current number of planet detections with M-star hosts is still low, due to the faintness of the targets in the visible wavelength range and the intrinsic stellar jitter. The CARMENES 
project (see also Quirrenbach et al. 2010, 2012) is aimed at filling this gap, by constructing a radial-velocity instrument optimized for planet searches of mid- to late-type $\mathrm{M}$ dwarfs.

\section{The CARMENES Instrument}

For mid-M to late-M spectral types, the wavelength range around $1000 \mathrm{~nm}$ ( $Y$ band) is the most important region for RV work. Therefore, the efficiency of CARMENES will be optimized in this range. Since CCDs do not provide high enough efficiency above $900 \mathrm{~nm}$, a near-IR detector is required. It is thus natural to adopt an instrument concept with two spectrographs, one equipped with a CCD for the range $550-1050 \mathrm{~nm}$, and one with $\mathrm{HgCdTe}$ detectors for the range from $0.9-1.7 \mu \mathrm{m}$.

Each spectrograph will be coupled to the $3.5 \mathrm{~m}$ telescope with its own optical fiber. The front end will contain a dichroic beam splitter and an atmospheric dispersion corrector, to feed the light into the fibers leading to the spectrographs. Additional fibers are available for simultaneous injection of light from emission line lamps for RV calibration.

The spectrographs are mounted on benches inside vacuum tanks, which are located in climatic chambers inside the coudé laboratory of the $3.5 \mathrm{~m}$ dome. The near-IR vacuum tank is equipped with a temperature stabilization system; the thermal design of both spectrographs provides the capability of keeping the temperature of the optical bench constant to within $\pm 0.01 \mathrm{~K}$ over $24 \mathrm{~h}$. The visible-light spectrograph will be operated near room temperature, the NIR spectrograph will be cooled to $\sim 140 \mathrm{~K}$.

\section{The CARMENES Survey}

The main scientific driver for CARMENES is the search for very low-mass planets (i.e., Earth-analogs and "super-Earths") around mid- to late-type M dwarfs. A long-term RV precision of $1 \mathrm{~m} \mathrm{~s}^{-1}$ is sufficient to detect a $2 M_{\oplus}$ planet in the middle of the $\mathrm{HZ}$ of an M5 star. For stars later than $\sim \mathrm{M} 4\left(M<0.25 M_{\odot}\right)$, such precision will yield detections of super-Earths of $5 M_{\oplus}$ and smaller inside the entire width of the HZ.

Our survey strategy is to intensively monitor a well-characterized sample of $\sim 300$ $\mathrm{M}$ dwarfs. If we assume that about $30-40 \%$ of the $\mathrm{M}$ dwarfs have low-mass planets and a high chance of some being in the habitable zone, we may expect to find between 50 and 100 suitable planets. Even if the real frequency is much lower, still a substantial number of detections will be available, and CARMENES will put tight constraints on the abundance and orbital parameters of low-mass planets in the investigated mass regime.

The expected CARMENES planet harvest is, at any rate, sufficient to carry out a reliable statistical analysis of the planet population and shed light on the architecture of planetary systems and on their formation mechanisms. The CARMENES survey will also provide valuable constraints on $\eta_{\oplus}$ for $\mathrm{M}$ dwarfs. Given the transit probability and the favorable selection bias, there is a good chance of finding 1 or 2 transiting low-mass planets in the habitable zone. These hold extraordinary value for future investigations.

\section{References}

Quirrenbach, A., Amado, P. J., Mandel, H., et al. (2010). CARMENES: Calar Alto highResolution search for $M$ dwarfs with Exo-earths with Near-infrared and optical Echelle Spectrographs. In Ground-based and airborne instrumentation for astronomy III. Eds. McLean, I. S., Ramsay, S. K., \& Takami, H., SPIE 773513, p. 1-14

Quirrenbach, A., Amado, P. J., Seifert, W., et al. (2012). CARMENES. I: Instrument and survey overview. In Ground-based and airborne instrumentation for astronomy IV. Eds. McLean, I. S., Ramsay, S. K., \& Takami, H., SPIE 84460R, p. 1-13 